\section{PALB2/FANCN, acteur dans la prédisposition au cancer du sein?}

Nancy Hamel, Marc Tischkowitz, William D. Foulkes
> Moins de $30 \%$ des cancers du sein héréditaires sont dus à des mutations dans des gènes connus dont la plupart participent aux processus de réparation de I'ADN (par exemple, BRCA1, BRCA2, TP53, ATM, CHK2) [1]. D'autres gènes participant à ce processus constituent donc des cibles potentielles pour des mutations prédisposant au développement du cancer du sein. Le gène PALB2, situé dans la région $16 \mathrm{p} 12.1$, a été récemment identifié en tant que partenaire du gène BRCA2 et est nécessaire à l'ancrage de BRCA2 aux structures nucléaires, permettant ainsi à BRCA2 de jouer son rôle dans le processus de réparation de I'ADN double-brin par recombinaison homologue [2]. Par ailleurs, des études récentes montrent que les mutations bialléliques dans PALB2, également appelé FANCN, causent l'anémie de Fanconi et produisent un phénotype très similaire à celui résultant des mutations bialléliques dans BRCA2 [3, 4]. Ces observations suggèrent que des mutations monoalléliques dans PALB2 pourraient prédisposer au cancer du sein comme celles qui surgissent dans BRCA2. Récemment, Rahman et al. ont identifié cinq mutations troncatrices monoalléliques dans PALB2 chez 10 femmes provenant d'une cohorte de 923 individus comptant une histoire familiale de cancer du sein. Les auteurs ont estimé que ces mutations augmentent le risque de cancer du sein d'un facteur 2,3 [5]. Parallèlement, une mutation fondatrice dans PALB2 a été décelée dans la population finlandaise et associée à une augmentation de risque d'un facteur 4 chez les individus porteurs [6] ; ces études pointent PALB2 comme un élément important dans la prédisposition au cancer du sein.

Nous avons séquencé PALB2 chez 119 probants issus de familles dont certains membres ont été victimes, soit de cancer du sein, soit de cancer de la prostate; sur ces 119 personnes, 84 proviennent d'une population fondatrice, soit canadienne-française, soit juive ashkénaze [7]. Notre analyse n'a identifié qu'une seule nouvelle mutation, 299delT (position 299 dans la séquence formée de nucléotides), chez une patiente d'ethnicité mixte atteinte d'un cancer du sein. Aucune mutation n'a été observée chez les Canadiens français ou les Juifs ashkénazes. La mutation 229 delT et la délétion 2521delA, décrites par Reid et al. [3], ont été introduites dans la séquence du gène $P A L B 2$ par mutagenèse ponctuelle dirigée et transfectées dans des cellules où les protéines mutantes ont été exprimées sous forme de protéines de fusion pour évaluer leur fonctionnalité (Figure 1). Les résultats montrent clairement que les deux protéines de fusion PALB2 mutées sont incapables, non seulement de s'attacher à BRCA2 comme le fait la protéine sauvage, mais également d'effectuer la réparation de I'ADN double-brin par recombinaison homologue.

Cependant, nous ignorons toujours si la présence de ces mutations à l'état hétérozygote affecte réellement les fonctions de réparation d'ADN chez les porteurs lorsqu'une copie non mutée demeure présente dans leurs cellules. Pour répondre à cette question, nous avons directement analysé les tumeurs des patients porteurs de ces deux muta-
M. Tischkowitz, W.D. Foulkes : Programme

en Génétique du cancer, Départements d'oncologie et de génétique humaine, Centre du cancer Ségal, Sir M.B. Davis, Hôpital Général Juif,

Montréal, Québec, Canada.

N. Hamel, W.D. Foulkes : Programme en Génétique du cancer, Départements d'oncologie et de génétique humaine, Institut de recherche du Centre universitaire de santé McGill,

1625, avenue des Pins, bureau L3-401, Montréal (Québec), H3G IA4 Canada.

nancy.hamel@mcgill.ca

tions. Comme dans le cas de BRCA2, les tumeurs PALB2 expriment les récepteurs des œstrogènes et de la progestérone sous forme de protéine. L'hybridation comparative sur puces à ADN génomique total (CGH-array) démontre que le profil du nombre de copies chromosomiques est généralement semblable pour les tumeurs liées à PALB2 et à BRCA2. En revanche, quelques différences existent. La perte chromosomique d'une partie de la région $18 q$ a été observée dans les 4 tumeurs malignes où PALB2 est muté mais pas dans les tumeurs où $B R C A 2$ est muté. De plus, une région couramment perdue sur $8 p$ dans les tumeurs $B R C A 1 / 2$, ainsi que dans les tumeurs de cancer du sein sporadiques n'ayant pas de mutations germinales, est préservée dans les tumeurs PALB2.

La perte d'hétérozygotie est un événement fréquemment observé dans la progression tumorale. C'est un phénomène par lequel l'allèle «normal » d'une tumeur hétérozygote pour une mutation est perdu ou inactivé, laissant l'allèle muté comme seule source d'expression pour la protéine, contribuant ainsi à la progression tumorale. Toutefois, notre analyse CGH-array n'a permis de détecter aucune perte chromosomique dans la région $16 p$ dans les tumeurs PALB2, et la comparaison de séquences incluant 
chacune de nos mutations démontre que l'allèle normal et l'allèle muté sont tous deux présents en quantité comparable dans les tumeurs PALB2. La perte d'hétérozygotie ne semble donc pas être un mécanisme lié à la progression de ces tumeurs; il n'est toutefois pas exclu que la copie «normale» de PALB2 dans ces tumeurs soit inactivée par une mutation ponctuelle située ailleurs dans la séquence du gène.

Les résultats à ce jour indiquent que PALB2 peut être responsable d'une proportion modeste de cas héréditaires de cancer du sein (environ 1,5\%) [5-7]. De plus, la présence de mutations fondatrices fréquentes dans PALB2 chez les Canadiens français ou les Juifs ashké- nazes, deux populations où de telles mutations dans BRCA2 sont présentes et bien caractérisées, ne semble pas probable. En revanche, la très forte histoire familiale de cancer du sein associée à la mutation 299 delT, la mutation la plus rapprochée de la position 5' du gène observée jusqu'à maintenant, suggère la possibilité d'une corrélation génotype-phénotype et l'existence de mutations conférant un risque plus élevé que celui précédemment décrit [5]. L'analyse CGH-array révèle que malgré de nombreuses similarités, il existe tout de même des différences moléculaires entre les tumeurs PALB2 et les tumeurs BRCA2 qui pourraient se traduire par différents mécanismes de

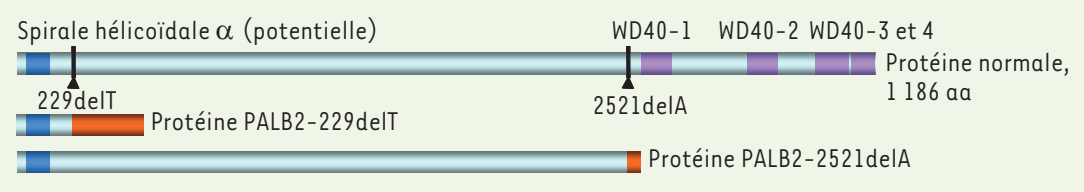

Figure 1. Conséquences des mutations 229delT et 2521delA sur la structure de PALB2. Les zones grises représentent les régions formées des acides aminés normaux de la protéine. La position des mutations respectives, à partir desquelles le code de lecture est changé, est indiquée sur la protéine sauvage, et les régions constituées de nouveaux acides aminés créés par le changement au code de lecture sont représentées en orangé pour chaque protéine mutante. On peut voir que la protéine mutée dérivée de la mutation 229 delT, en particulier, possède une longue série de 99 nouveaux acides aminés non présents dans la protéine sauvage. Les 4 motifs WD40 (en mauve sur la protéine sauvage) servant aux interactions protéine-protéine sont manquants, ce qui signifie que si ces protéines sont produites dans les cellules des porteurs, elles ont sans doute perdu la capacité d'interagir avec bon nombre des partenaires normaux de PALB2. II est à noter qu'un motif spirale hélicoïdale de type alpha est potentiellement situé dans la partie amino-terminale de la protéine sauvage (en bleu) et est conservé dans les deux protéines de fusion et pourrait également favoriser ce type d'interaction. Cependant, l'observation expérimentale qui établit que ces protéines mutantes de fusion ne sont plus capables de s'attacher à BRCA2 suggère que ce sont les motifs WD40 qui sont nécessaires pour cette interaction. progression tumorale chez les porteurs respectifs. Le processus exact par lequel une mutation monoallélique dans PALB2 peut induire la formation d'un cancer du sein demeure inconnu; cependant une perte de fonction totale ou un effet dominant négatif inhibant la réparation d'ADN sont peu probables. Une autre possibilité serait que PALB2 soit un gène suppresseur de tumeur haploinsuffisant; l'étroite relation entre les fonctions de PALB2 et de BRCA2 pourrait être particulièrement sensible aux effets de dosage. La détection et la caractérisation de mutations additionnelles dans le gène $P A L B 2$, ainsi que des analyses biochimiques de cette protéine seront des facteurs clés pour mieux comprendre les mécanismes moléculaires en cause dans la progression de ces tumeurs. $\diamond$ A role for PALB2/FANCN in breast cancer predisposition?

\section{RÉFÉRENCES}

1. Wooster R, Weber BL. Breast and ovarian cancer. $N$ Engl J Med 2003; 348 : 2339-47.

2. Xia $B$, Sheng $Q$, Nakanishi $K$, et al. Control of BRCA2 cellular and clinical functions by a nuclear partner, PALB2. Mol Cell $2006 ; 22$ : 719-29.

3. Reid S, Schindler D, Hanenberg H, et al. Biallelic mutations in PALB2 cause Fanconi anemia subtype FA-N and predispose to childhood cancer. Nat Genet 2007 ; 39 : 162-4.

4. Xia B, Dorsman JC, Ameziane N, et al. Fanconi anemia is associated with a defect in the BRCA2 partner PALB2. Nat Genet 2007 ; 39 : 159-61.

5. Rahman N, Seal S, Thompson D, et al. PALB2, which encodes a BRCA2-interacting protein, is a breast cancer susceptibility gene. Nat Genet 2007 ; 39: 165-7.

6. Erkko $\mathrm{H}, \mathrm{Xia} B$, Nikkilä J, et al. A recurrent mutation in PALB2 in Finnish cancer families. Nature 2007; 446 : 316-9.

7. Tischkowitz M, Xia B, Sabbaghian N, et al. Analysis of PALB2/FANCN-associated breast cancer families. Proc Natl Acad Sci USA 2007 ; 104 : 6788-93. 